\title{
Liver injury is most commonly due to hepatic metastases rather than drug hepatotoxicity during pembrolizumab immunotherapy
}

\author{
Irene Tsung ${ }^{1}$ | Russell Dolan ${ }^{1}$ | Christopher D. Lao ${ }^{2}$ | Leslie Fecher ${ }^{2}$ | \\ Kane Riggenbach $^{1}$ | Amoah Yeboah-Korang ${ }^{3}$ | Robert J. Fontana ${ }^{3}$ (D)
}

${ }^{1}$ Department of Internal

Medicine, University of Michigan, Ann

Arbor, Michigan

${ }^{2}$ Division of Hematology and

Oncology, University of Michigan, Ann

Arbor, Michigan

${ }^{3}$ Division of Gastroenterology and Hepatology, University of Michigan, Ann Arbor, Michigan

\section{Correspondence}

Dr. Robert J. Fontana, Division of Gastroenterology and Hepatology, University of Michigan, Ann Arbor, MI.

Email: rfontana@umich.edu

\section{Summary}

Background: Pembrolizumab immunotherapy has been associated with hepatotoxicity in $1 \%-10 \%$ of oncology patients treated in clinical trials.

Aim: To describe the incidence, phenotypes and outcomes of liver injury in a large cohort of solid organ tumour patients receiving pembrolizumab

Methods: Liver injury was defined by serum alanine aminotransferase, alkaline phosphatase, and/or total bilirubin levels exceeding threshold values. The likelihood of drug-induced liver injury was adjudicated by expert opinion.

Results: Seventy (14.3\%) of the 491 pembrolizumab-treated patients developed liver injury at a median of 62 days (6-478) and $71.4 \%$ had a cholestatic injury profile at onset. The median age, gender and tumour types of liver injury patients were similar to those without, but hepatic metastases (53\% vs 21\%, $P<0.01$ ) and prior systemic and liver-directed therapy ( $71 \%$ vs $53 \%, P<0.01$ ) were more commonly observed in liver injury patients. During follow-up, liver injury patients were less likely to experience tumour remission ( $10 \%$ vs $40.4 \%$ ) and had higher mortality (67.1\% vs $33.7 \%$ ). Only 20 (28.6\%) liver injury cases were adjudicated as probable drug-induced hepatotoxicity; these patients were significantly more likely to present with an hepatocellular/mixed injury pattern ( $65 \%$ vs $12 \%$ ), to receive corticosteroids (55\% vs $12 \%$ ) and had lower mortality (45\% vs $76 \%$ ) during follow-up.

Conclusions: Oncology patients treated with pembrolizumab who develop liver injury experience poorer outcomes during follow-up. The low incidence of confirmed drug hepatotoxicity highlights the need for thorough medical evaluation before initiating corticosteroids to optimise patient care. 


\section{1 | INTRODUCTION}

Treatment of advanced solid organs tumours has rapidly evolved in the past 10 years with the approval of over 60 new agents. ${ }^{1}$ Immune checkpoint inhibitors (ICI) that target the cell surface receptors of the programmed-death receptor ligand-1 (PD-1) and CTLA-4 invoke a regulated $T$-cell response against tumour cells by inhibiting internal T-cell checkpoints. ${ }^{2-5}$ In addition to improved tumour responses, use of these monoclonal antibodies has been associated with a systemic form of drug toxicity that has prominent autoimmune features commonly referred to as immune-related adverse events (irAE's) which can afflict many internal organs, ${ }^{6-8}$ including the liver in $1 \%-10 \%$ of patients treated in clinical trials. ${ }^{2,9,10}$ Although the definitions used to determine the incidence and severity of hepatotoxicity have been variable, most studies have demonstrated a higher incidence of liver injury in patients treated with high dose anti-CTLA-4 agents and with combination regimens of anti-CTLA- 4 and anti-PD1/ anti-PDL1 therapy. But the incidence, phenotype and risk factors for liver injury in patients receiving these drugs in clinical practice are not well described.

Pembrolizumab is an $\mathrm{ICl}$ administered as an intravenous infusion every 3 weeks that was initially approved for patients with advanced melanoma in $2014 .{ }^{11}$ Pembrolizumab is most commonly used in latestage cancer patients who likely harbour known or occult metastases. Oncology practice guidelines recommend that corticosteroids and other immunosuppressants be rapidly initiated in any patient receiving an $\mathrm{ICl}$ with an $\mathrm{ALT} \geq 3 \mathrm{x}$ upper limit of normal (ULN). ${ }^{9}$ However, the aetiology of these liver biochemistry abnormalities and the impact of developing liver injury on clinical outcomes are not well described. The aim of this study is to determine the incidence, aetiologies and outcomes of consecutive cancer patients with varying tumour types who developed liver injury during pembrolizumab immunotherapy at a large tertiary care cancer centre.

\section{2 | MATERIALS AND METHODS}

\section{1 | Data collection}

This retrospective study was approved by the Institutional Review Board of Michigan Medicine. Consecutive patients receiving pembrolizumab immunotherapy between 1 January 2014 and 1 January 2018 were identified from the Michigan Medicine Cancer Registry database. One patient was excluded due to enrolment in a doubleblind clinical trial yielding a total of 491 evaluable patients. Using Data Direct, a self-serve electronic medical record (EMR) search tool, patient health data were extracted including birthdate, sex, race, ethnicity, body mass index (BMI) and the dose and frequency of pembrolizumab infusions administered. In addition, serial serum aspartate aminotransferase (AST), alanine aminotransferase (ALT), alkaline phosphatase (ALP), total bilirubin and international normalised ratio (INR) levels prior to, during, and after immunotherapy were reviewed. Available diagnostic test results in patients with liver injury including serum antinuclear antibody (ANA), smooth muscle antibody (SMA), hepatitis A, B and C serologies and results of liver imaging were extracted. All pembrolizumab doses were verified through chart review. Manual EMR review was used to record additional data including prior treatment with other immunotherapy or liver-directed therapy within the prior year, tumour type, pre-treatment liver imaging, liver biopsy reports, management of adverse events including use of steroids, tumour response and date and cause of death.

\subsection{Definition of liver injury and adjudication of aetiology}

Baseline liver biochemistries of serum AST, ALT, ALP and total bilirubin were defined as those results obtained immediately prior to the first pembrolizumab dose. Liver injury on treatment was defined using the Drug-Induced Liver Injury Network (DILIN) study criteria ${ }^{12}$ as any of the following within 90 days of the last pembrolizumab infusion:

1. Serum ALT $\geq 5 x$ upper limit of normal (or baseline if baseline > ULN)

2. Serum ALP $\geq 2 x$ ULN (or baseline if baseline $>$ ULN)

3. Total bilirubin $\geq 2.5 \mathrm{mg} / \mathrm{dL}$ (or $>2 x$ baseline if baseline $>U L N$ )

The peak liver biochemistry test results were recorded as the maximum values after meeting liver injury criteria; normalisation of liver biochemistries was determined when the abnormal lab test returned to less than ULN. Four physician reviewers (IT, RD, RJF, AYK) adjudicated each liver injury case to determine the likelihood of drug-induced hepatotoxicity versus an alternative aetiology and assigned each case an expert opinion DILIN causality score ranging from 1 = definite, 2 = highly likely, 3 = probable, 4 = possible, and $5=$ Unlikely. ${ }^{12}$ In addition, $R$ values were calculated using the equation $R=$ (serum ALT/ULN)/ (ALP/ULN) and classified as hepatocellular $(R>5)$, mixed $(R=2-5)$, or cholestatic $(R<2)$. The Roussel Uclaf Causality Assessment Method $(R \cup C A M)^{13}$ was calculated for each of the liver injury cases and compared with the expert opinion adjudication score. Total RUCAM scores vary from -9 to +14 and are categorised as $\leq 0=$ drug excluded as a cause; 1 to 2 = unlikely; 3 to $5=$ possible; 6 to $8=$ probably; $>8=$ highly probable.

\subsection{Statistical analysis}

Descriptive statistics were calculated using mean (standard deviation) or median (range) for normally and nonnormally distributed data, respectively. Kaplan-Meier survival curves were calculated from the time of pembrolizumab infusion to death or last available follow-up in patients with and without liver injury. Baseline features associated with new onset liver injury were determined using univariate and multivariate models. Statistical analyses were performed using the Chi-squared, Mann-Whitney $U$, and Kruskal-Wallis tests. 


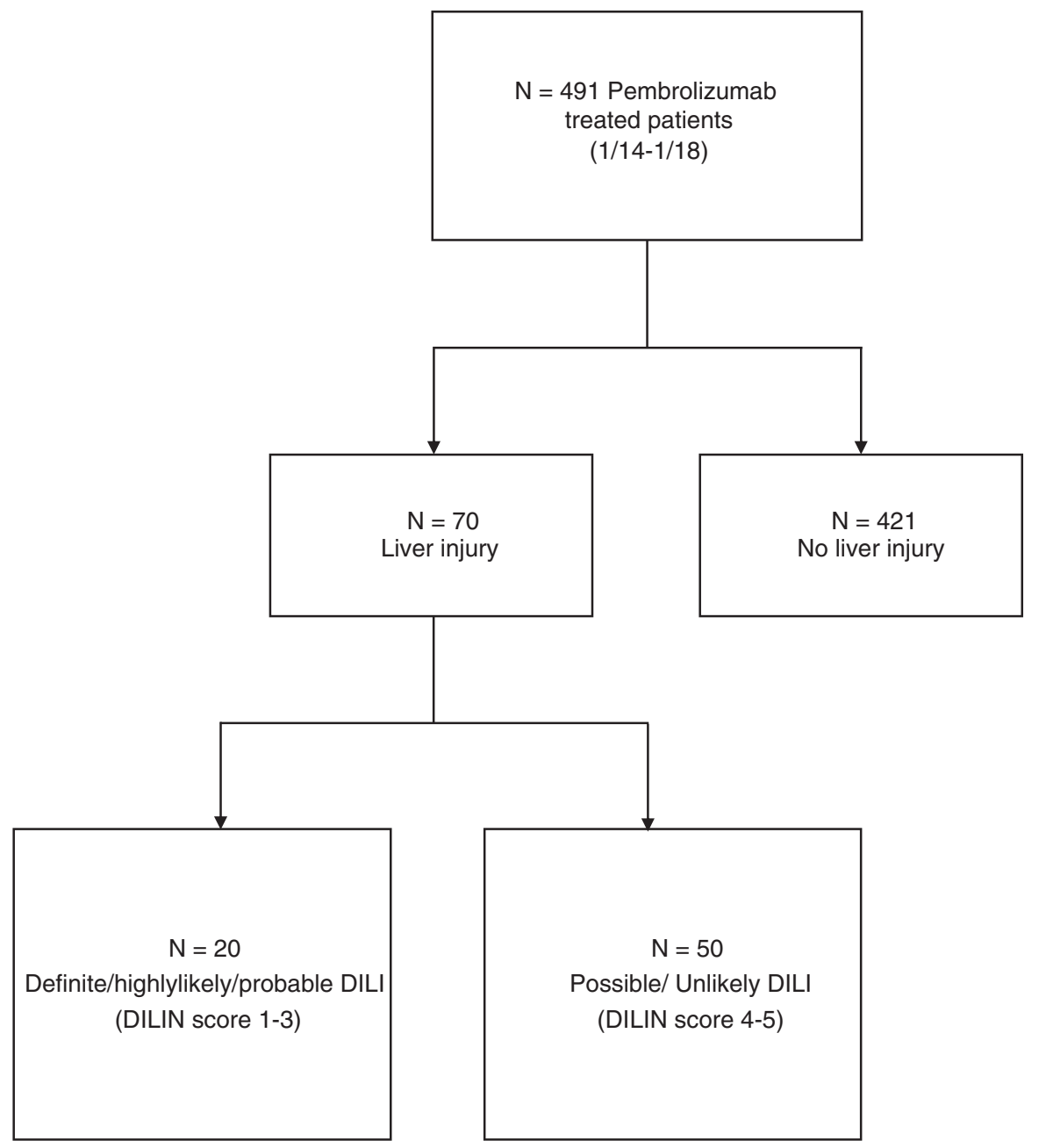

FIGURE 1 Patient population and flowchart. There were 491 patients who received pembrolizumab treatment for cancer. During treatment, 70 patients met laboratory criteria for liver injury while the remaining 421 patients did not. Following expert opinion adjudication, only 20 (28\%) of the liver injury cases were attributed to a medication
Statistical significance was defined as $P<0.05$. Analyses were completed in RStudio statistical software (Boston, MA).

\section{3 | RESULTS}

\section{1 | Patient characteristics}

A total of 491 patients received pembrolizumab treatment between January 2014 and January 2018. During a median follow-up of 211 days, 70 (14\%) patients met the predefined laboratory criteria for liver injury (Figure 1). The liver injury was predominantly cholestatic (71.4\%) at onset with only $17.1 \%$ having a total bilirubin $>2.5 \mathrm{mg} / \mathrm{dL}$. The specific lab criteria that were met included serum AST or ALT elevations in 12 (17.1\%), ALP elevations in 42 (60\%), total bilirubin elevations in eight (11.4\%), ALP and ALT elevations in four (5.7\%), ALP and total bilirubin in two (2.8\%), and all three criteria in two (2.8\%). Most of the liver injury cases (61\%) occurred within the first 3 months of treatment and $77 \%$ occurred within the first 6 months of treatment. The median number of pembrolizumab infusions received in those with and without liver injury was significantly lower ( 3 vs $5, P<0.01$ ). The proportion of pembrolizumab-treated patients who developed liver injury did not substantially change over time (data not shown).

The median age, race and BMI of the 70 patients with liver injury were similar to the 421 without liver injury (Table 1 ). The types and stage of solid organ tumours were also similar in the two groups with $65 \%$ of the cohort having either metastatic melanoma, lung or urothelial cancer. However, the patients with liver injury were significantly more likely to have received prior chemotherapy or liverdirected locoregional therapy in the year prior to pembrolizumab (71.3\% vs $53 \%, P<0.01)$ and to have known hepatic metastases prior to treatment (52.9\% vs $21.4 \%, P<0.001)$. Interestingly, the presence of hepatic steatosis on imaging and the pre-treatment serum ALT and total bilirubin levels were similar in patients with and without liver injury but the pre-treatment serum AST and ALP levels were both significantly higher in the liver injury group. During follow-up, a significantly lower rate of tumour remission (10\% vs $40.4 \%$ ) was observed in those with liver injury as well as a significantly lower actuarial patient survival (33.7\% vs $67.1 \%$ ) compared to the 421 patients without liver injury (Figure 2). Finally, the frequency of nonhepatic irAE was similar in those with and without liver injury (15\% vs $21 \%$ ) suggesting that the risk of developing liver injury was independent of other adverse events. 
TAB LE 1 Clinical characteristics of the study population

\begin{tabular}{|c|c|c|c|}
\hline & $\begin{array}{l}\text { Liver injury } \\
N=70\end{array}$ & $\begin{array}{l}\text { No liver injury } \\
N=421\end{array}$ & $P$ value \\
\hline Age (y) & $64[16-89]$ & $65[16-97]$ & 0.71 \\
\hline BMI $\left(\mathrm{kg} / \mathrm{m}^{2}\right)$ & $28.4[14.6-44.7]$ & $27.2[14.8-49.7]$ & 0.57 \\
\hline \multicolumn{4}{|l|}{ Race } \\
\hline African American & $3(4.3)$ & $10(2.4)$ & \\
\hline Asian & $2(2.9)$ & $3(0.7)$ & \\
\hline Native Hawaiian/Other Pacific & $0(0.0)$ & $2(0.5)$ & \\
\hline American Indian or Alaska Native & $0(0.0)$ & $1(0.2)$ & \\
\hline Unknown & $2(2.9)$ & $6(1.4)$ & \\
\hline Unknown & $0(0.0)$ & $8(1.9)$ & \\
\hline Prior immunotherapy (ipilimumab, nivolumab) & $11(15.7)$ & $67(15.8)$ & 0.97 \\
\hline Total \# pembrolizumab infusions & $3[1-20]$ & $5[1-43]$ & 0.01 \\
\hline Duration of follow-up (d) & $142.4[2.3-1146.3]$ & $230.4(2.4-1159.4]$ & 0.12 \\
\hline \multicolumn{4}{|l|}{ Cancer type } \\
\hline Melanoma & $30(42.9)$ & $182(43.2)$ & 0.95 \\
\hline Lung & $5(7.1)$ & $60(14.3)$ & \\
\hline Urothelial & $11(15.7)$ & $35(8.2)$ & \\
\hline AST (IU/L) & 28 [12-213] & 24 [11-179] & 0.02 \\
\hline ALT (IU/L) & $22[8-83]$ & 21 [8-173] & 0.72 \\
\hline Alkaline phosphatase (IU/L) & $108[43-523]$ & $90[26-1147]$ & $<0.01$ \\
\hline Total bilirubin (IU/L) & $0.5[0.1-1.1]$ & $0.4[0.1-8.3]$ & 0.72 \\
\hline Tumour outcome through 1/1/18 & & & $<0.01$ \\
\hline Progression & $16(22.9)$ & $109(25.9)$ & \\
\hline Stable/ remission & $7(10)$ & $170(40.4)$ & \\
\hline Death & $47(67.1)$ & $142(33.7)$ & \\
\hline
\end{tabular}

Note: Data presented as median [range] or $\mathrm{n}(\%)$.

Abbreviations: ALT, alanine aminotransferase; AST, aspartate aminotransferase; BMI, body mass index; irAE, immune-related adverse event; TACE, transarterial chemoembolisation; XRT, radiotherapy.

\subsection{Factors associated with development of liver injury}

Variables which demonstrated a $P$ value of $<0.10$ (Table 1) were entered into univariate and multivariate logistic regression models of new onset liver injury. After adjusting for subject gender, pretreatment AST and ALP, and prior liver-directed therapy, only the presence of hepatic metastases was an independent predictor of liver injury (Odds ratio: 3.58, 95\% Cl: 2.03-6.31, $P<0.01$ ). (Table S1).

\section{3 | Expert adjudication of the 70 liver injury cases}

All of the available laboratory, radiological and clinical data of the 70 patients with liver injury were reviewed and scored by the investigators 
using the DILIN expert opinion scale of 1 (definite) to 5 (unlikely). ${ }^{12}$ There were one definite, six highly likely and 13 probable drug-induced hepatotoxicity cases while the remaining 50 cases were adjudicated as possible (21) or unlikely (29) cases of drug hepatotoxicity (Table 2). The $20(28.6 \%)$ patients with a causality score of 1,2 or 3 represented $4.1 \%$ of all of the 491 patients treated with pembrolizumab. However, three of these high causality cases were attributed to a drug other than pembrolizumab (ipilimumab, vemurafenib and dabrafenib) but were analysed with the pembrolizumab hepatotoxicity cases.

The median number of pembrolizumab infusions prior to liver injury onset was similar in the high and low causality cases ( 3 vs 3 , $P=0.63$ ), and the time to liver injury onset was also similar (66 vs 62 days, $P=0.56$ ). In addition, the baseline patient demographic features and pre-treatment liver biochemistries were similar in the high versus low causality liver injury cases. However, the 20 patients with high causality scores were more likely to have an acute hepatocellular or mixed liver injury at onset compared to the 50 low causality patients ( $65 \%$ vs $12 \%, P<0.01$ ). In addition, the high causality cases had significantly higher peak serum ALT levels but the proportion with jaundice was similar (Table 2). The most commonly identified alternative cause of liver injury among the 50 possible/unlikely cases was progressive hepatic tumour metastases (56\%), while other aetiologies included malignant biliary obstruction (4\%), nonhepatic disease (9\%) and other biliary obstruction or unknown (2\%).

\subsection{RUCAM scoring of the 70 liver injury cases}

The RUCAM scores were significantly higher in the 20 high causality cases compared to the low causality cases (5 vs 2). The overall level of concordance between the RUCAM and DILIN expert opinion scales was relatively good with a Pearson correlation coefficient of -0.57 (Figure S1).

\subsection{Clinical outcomes in the 70 patients with liver injury}

The median duration of follow-up in the low causality cases was significantly shorter (122 vs 333 days, $P<0.01$ ) largely due to the higher observed mortality during follow-up in the low causality cases ( $76 \%$ vs $45 \%, P=0.01$ ) (Figure 3 ). The adjudicated causes of death were tumour progression in seven of the nine high causality patients who died and tumour progression in 31 of the 38 low causality patients who died. None of the nine deaths in the high causality group were directly attributed to pembrolizumab drug hepatotoxicity. The higher mortality in the low causality group may have, in part, been due to the higher incidence of hepatic metastases on pre-treatment imaging ( $66 \%$ vs $20 \%$ ) as well as the lower rate of objective tumour stabilisation/remission during follow-up (4\% vs $25 \%$ ).

The high causality patients were significantly more likely to receive corticosteroids after liver injury onset (Table 2). They also experienced more frequent normalisation of liver biochemistry abnormalities during follow-up. Of note, none of the high causality

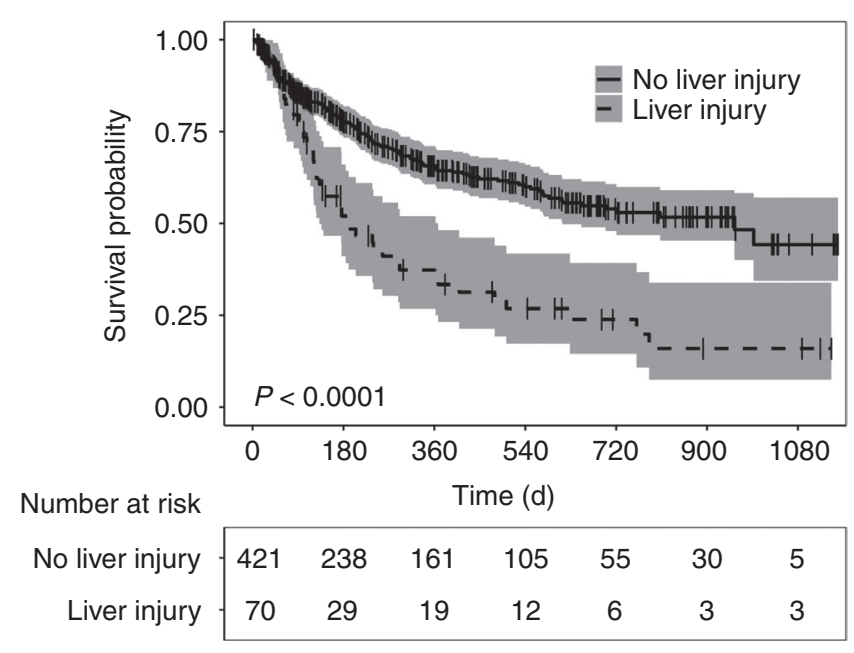

FIGURE 2 Actuarial patient survival after starting pembrolizumab treatment. The overall patient survival in the 70 patients who developed liver injury was significantly lower compared to the 421 without liver injury during follow-up $(P<0.0001$ using Kaplan-Meier statistics $)$

case patients were re-challenged with pembrolizumab. A review of the available liver pathology in four of the high causality patients who underwent biopsy at a median of 14 days after liver injury onset demonstrated a variety of histological findings (Table S2). However, none of these patients had eosinophils, granulomas or plasma cells noted on biopsy nor detectable serum autoantibodies.

\subsection{Analysis of 70 liver injury cases stratified by liver injury pattern}

The 70 liver injury cases were stratified by the biochemical pattern of liver injury at onset (ie hepatocellular, cholestatic, mixed) as defined by the $\mathrm{R}$ ratio (Table S3). There were 11 patients who presented with an acute hepatocellular injury profile, 51 with a cholestatic profile, and eight with a mixed profile. Variables with significant differences between groups are similar to the results obtained when stratifying the 70 liver injury patients by DILIN expert opinion score. There were significant differences in the frequency of baseline hepatic metastases, hepatic steatosis and pre-treatment serum AST and ALT levels. In addition, those with an acute hepatocellular or mixed injury were more likely to receive corticosteroids and normalise their liver biochemical abnormalities during follow-up. Finally, subjects with a cholestatic liver injury profile also had a poorer prognosis during follow-up than those presenting with a hepatocellular or mixed laboratory profile.

\section{4 | DISCUSSION}

The onset of liver biochemical abnormalities at 3-6 months after starting treatment is a well recognised side effect of checkpoint inhibitor immunotherapy. ${ }^{2}$ Grading schemas largely based upon the level of serum ALT elevation have been developed 
TAB LE 2 Liver injury cases stratified by DILIN causality score

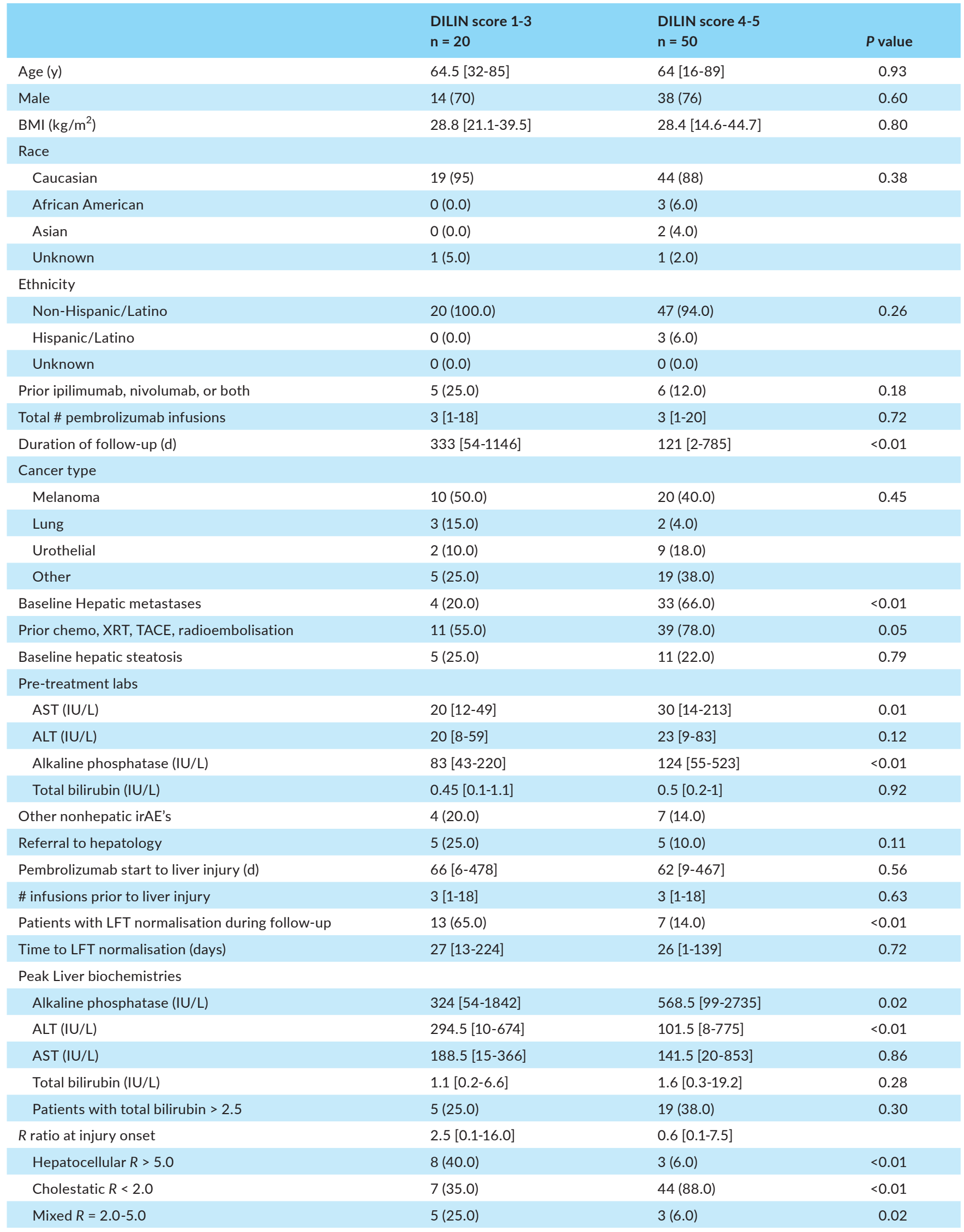


TABLE 2 (Continued)

\begin{tabular}{|c|c|c|c|}
\hline & $\begin{array}{l}\text { DILIN score } 1-3 \\
n=20\end{array}$ & $\begin{array}{l}\text { DILIN score } 4-5 \\
n=50\end{array}$ & $P$ value \\
\hline RUCAM & $5[1-9]$ & $2[0-9]$ & $<0.01$ \\
\hline Patients with pembrolizumab discontinued after liver injury & $17(85.0)$ & $39(78.0)$ & 0.51 \\
\hline Tumour outcome through $1 / 1 / 18$ & & & $<0.01$ \\
\hline Stable/Remission & $5(25.0)$ & $2(4.0)$ & \\
\hline Death & $9(45.0)$ & $38(76.0)$ & \\
\hline
\end{tabular}

Note: Data presented as median [range] or $\mathrm{n}(\%)$.

Abbreviations: ALT, alanine aminotransferase; AST; aspartate aminotransferase; BMI, body mass index; irAE, immune-related adverse event; LFT, liver function test; RUCAM, Roussel Uclaf Causality Assessment; TACE, transarterial chemoembolisation; XRT, radiotherapy.



FIG URE 3 Actuarial patient survival in 70 liver injury patients. The 20 patients with probable drug-induced hepatotoxicity (DILIN score of $1,2,3$ ) had a significantly better survival compared to the 50 patients with other causes of liver injury (DILIN score 4, 5) $(P<0.0005$ using Kaplan-Meier statistics $)$

and incorporated into clinical practice guidelines as well as recommendations for the evaluation of other potential causes. ${ }^{14}$ However, the risk factors and outcomes in patients who develop any form of biochemical liver injury during checkpoint inhibitor immunotherapy remain poorly understood. In this study of 491 consecutive patients receiving pembrolizumab, liver injury occurred in 70 (14\%) patients. In general, liver injury was predominantly cholestatic $(R$ ratio $<2.0$ ) and mild in severity at onset and the baseline demographic and laboratory features of the patients with liver injury versus those without liver injury were similar (Table 1). However, patients who experienced liver injury had a significantly lower tumour response and a higher mortality during follow-up (Figure 2). The poorer outcomes in the liver injury group may relate to the larger proportion with baseline hepatic metastases and other recent treatments prior to pembrolizumab that is consistent with more advanced and refractory cancer. On multivariate analysis, pre-treatment hepatic metastases were the only independent baseline factor associated with the development of liver injury (Table S1).

Only a minority of the liver injury cases were attributed to pembrolizumab hepatotoxicity (29\%) while cancerous replacement of the liver accounted for most of the other patients with benign or malignant biliary obstruction identified in $5.7 \%$. (Table 2). These data are important since most prior publications on this topic have simply described the incidence of liver injury during treatment but not adjudicated the cause. ${ }^{8,15}$ Furthermore, current treatment guidelines recommend the rapid institution of corticosteroids and withholding of further treatment whenever the serum ALT exceeds $3 x$ ULN or ALP increases to more than $2 x$ ULN. ${ }^{9}$ However, our data indicate that careful clinical assessment of the cause of liver injury is critical including contrast enhanced cross-sectional imaging of the liver to assess for tumour progression and to help ensure that the appropriate actions are undertaken. ${ }^{16}$ If other prospective studies confirm our observations, less frequent use of immunosuppression may be indicated and additional cancer treatments could be offered to improve patient outcomes. In addition, prospectively obtained liver biopsies in patients with elevated liver biochemistries may prove informative to help guide management. Our data demonstrate that patients who present with an acute hepatocellular or mixed injury pattern are more likely to be experiencing immune-mediated liver injury due to pembrolizumab but there was no particular serum ALT, ALP, or bilirubin level that reliably differentiated patients with DILI from other causes of liver injury (Table S3).

The 20 patients with drug hepatotoxicity (DILIN score of 1-3) were more likely to have a hepatocellular or mixed injury at presentation compared to the 50 with low causality scores (65\% vs $12 \%$ ). In addition, these patients had higher peak serum ALT levels and were more likely to receive corticosteroids and experience liver biochemistry normalisation during follow-up suggesting that their liver injury was indeed immune-mediated and steroid-responsive (Table 2). Unfortunately, results of serum autoantibodies and quantitative immunoglobulin levels were available in only four of the 20 high causality cases. This may be due to the fact that only a small proportion of the liver injury patients (14\%) were seen by a GI or liver specialist and even fewer (7\%) underwent a liver biopsy. Interestingly, the 20 high 
causality drug hepatotoxicity patients had significantly better shortterm survival compared to the 50 patients with other causes of liver injury (Figure 3). This is likely due to the fact that the high causality cases were more likely to experience an objective tumour response while the majority of low causality cases had hepatic metastases that progressed during follow-up. Analysis of our data based upon the Rvalue at liver injury onset showed similar results with hepatocellular and mixed injury patients being more likely to have a higher causality score, receive corticosteroids and normalise their labs during followup compared to those with a cholestatic profile (Table S3).

Information regarding the liver histology in patients with immunotherapy hepatotoxicity is not well described. Kleiner et al originally described 10 patients with moderately severe acute hepatocellular injury wherein many had pericentral necrosis or plasma cell-mediated hepatitis. ${ }^{17}$ In a more recent multicentre French study, fibrin ring granulomas, hepatic steatosis and periportal hepatitis were more commonly identified than plasma cell hepatitis particularly in patients receiving anti-CTLA-4 therapy. ${ }^{18}$ In the current study, four high causality patients underwent a liver biopsy at a median of 13 days (range 1-45) after DILI onset but only mild apoptosis and hepatic steatosis were observed (Table S2). Therefore, our data are consistent prior reports demonstrating a spectrum of liver histopathological abnormalities in patients with pembrolizumab hepatotoxicity. ${ }^{19}$ Whether the pattern or severity of liver pathology findings correlates with response to corticosteroids or has independent prognostic value requires further study in a larger number of patients undergoing biopsy.

Since expert opinion is not widely available, standardised RUCAM scores were also calculated for each of the 70 cases. Contrary to our expectations, there was a high degree of concordance between the RUCAM and expert opinion causality scores (Figure S1). ${ }^{20}$ Interestingly, very few of the cases achieved a very high RUCAM score (> 8) (8 cases) since most patients did not undergo testing for competing causes of liver injury.

Strengths of our study include the large number of patients (ie 491) who received pembrolizumab treatment at a single centre over a 4-year period. Furthermore, these therapies were given by a limited number of oncologists in a standardised manner. In general, the dose of pembrolizumab was $2 \mathrm{mg} / \mathrm{kg}$ or $200 \mathrm{mg}$ flat dose given every 3 weeks with laboratory monitoring obtained prior to each infusion. However, the criteria to withhold an infusion of pembrolizumab due to hepatotoxicity and when and how much corticosteroids to use were variable. Additionally, our EMR database did not allow us to determine the cumulative corticosteroid dose administered. However, all of the liver injury cases were vetted for causality using an established expert opinion method as well as the RUCAM. The high degree of concordance in these two scales suggests that the causality assessment used in this study was robust and reproducible. A final limitation of our study was its retrospective nature and the lack of diagnostic serologies for competing causes of liver injury in many of the liver injury cases. However, nearly $80 \%$ of the patients did undergo contrast enhanced liver imaging after liver injury onset. Going forward, future prospective studies should include a more comprehensive assessment for competing aetiologies of liver injury in patients receiving checkpoint inhibitors with hepatotoxicity. However, the value of routine assessment of baseline and on treatment autoantibodies remains controversial since prior studies have demonstrated a low incidence of discriminating high titre autoantibodies in patients with bonafide hepatotoxicity. ${ }^{2,17,21}$

In conclusion, the overall incidence of liver injury observed in our study of $14 \%$ is somewhat higher than that reported in licensing trials and other series. ${ }^{2,22}$ However, the liver injury patients had a significantly poorer short-term survival (Figure 2). Since the majority of deaths observed was not due to drug-induced hepatotoxicity, our data suggest that the poorer prognosis is likely due to the presence of more advanced cancer and liver metastases prior to treatment rather than hepatotoxicity per se from pembrolizumab therapy. (Figure 3). Going forward, pembrolizumab-treated patients who develop liver biochemical abnormalities should undergo thorough evaluation for competing causes of liver injury and liver biopsy, whenever feasible, to assist with diagnosis and guide the appropriate use of immunosuppressive therapy rather than empiric therapy in all patients.

\section{ACKNOWLEDGEMENTS}

Declaration of personal interests: IT, RD, KR AY and CL have no disclosures. RJF receives grant support from Abbvie, BMS, and Gilead and does consulting for Sanofi. LF receives grant support from Merck, Incyte, BMS, EMD serono/ Pfizer and dose consulting for Via Oncology/ Elsevier and Hoosier Cancer Research Network.

\section{AUTHORSHIP}

\section{Guarantor of the article: None.}

Author contributions: IT, study design, data collection, analysis and manuscript drafting; RD, study design, data collection, analysis and manuscript drafting; $\mathrm{CL}$, analysis and manuscript drafting; $\mathrm{LF}$, analysis and manuscript drafting; $\mathrm{KR}$, data analysis; $\mathrm{AY}-\mathrm{K}$, analysis and manuscript drafting; RF, study design, data collection, analysis and manuscript drafting.

\section{ORCID}

Robert J. Fontana (iD https://orcid.org/0000-0001-9161-5892

\section{REFERENCES}

1. Beaver JA, Howie LJ, Pelosof L, et al. A 25-year experience of US Food and drug administration accelerated approval of malignant hematology and oncology drugs and biologics: a review. JAMA Oncol. 2018;4:849-856.

2. Suzman DL, Pelosof L, Rosenberg A, Avigan MI. Hepatotoxicity of immune checkpoint inhibitors: an evolving picture of risk associated with a vital class of immunotherapy agents. Liver Int. 2018;38:976-987. 
3. Sharma P, Allison JP. The future of immune checkpoint therapy. Science. 2015;348:7.

4. Leach DR, Krummel MF, Allison JP. Enhancement of antitumor immunity by CTLA-4 blockade. Science. 1996;271:1734-1736.

5. Sharma P, Wagner K, Wolchok JD, Allison JP. Novel cancer immunotherapy agents with survival benefit: recent successes and next steps. Nat Rev Cancer. 2011;11:805-812.

6. Hassel JC, Heinzerling L, Aberle J, et al. Combined immune checkpoint blockade (anti-PD-1/anti-CTLA-4): evaluation and management of adverse drug reactions. Cancer Treat Rev. 2017;57:36-49.

7. Wang DY, Salem JE, Cohen JV, et al. Fatal toxic effects associated with immune checkpoint inhibitors: a systematic review and metaanalysis. JAMA Oncol. 2018;4:1721-1728.

8. Postow MA, Sidlow R, Hellmann MD. Immune-related adverse events associated with immune checkpoint blockade. N Engl J Med. 2018;378:158-168.

9. Haanen J, Carbonnel F, Robert C, etal. Management of toxicities from immunotherapy: ESMO Clinical Practice Guidelines for diagnosis, treatment and follow-up. Ann Oncol. 2017;28(suppl_4):iv119-iv142.

10. Wang W, Lie P, Guo M, He J. Risk of hepatotoxicity in cancer patients treated with immune checkpoint inhibitors: a systematic review and meta-analysis of published data. Int J Cancer. 2017;141:1018-1028.

11. Chuk MK, Chang JT, Theoret MR, et al. FDA approval summary: accelerated approval of pembrolizumab for second-line treatment of metastatic melanoma. Clin Cancer Res. 2017;23:5666-5670.

12. Fontana RJ, Watkins PB, Bonkovsky HL, et al. Drug induced liver injury network prospective study. Drug Saf. 2009;32:55-68.

13. Benichou C, Danan G, Flahault A. Causality assessment of adverse reactions to drugs-II. An original model for validation of drug causality assessment methods: case reports with positive rechallenge. J Clinical Epidemiology. 1993;46:1331-1336.

14. Brahmer J, Lacchetti C, Schneider BJ, et al. Management of immunerelated adverse events in patients treated with immune checkpoint inhibitor therapy: American Society of Clinical Oncology Practice Guideline. J Clin Oncol. 2018;36:1714-1768.

15. Huffman BM, Kottschade LA, Kamath PS, Markovic SN. Hepatotoxicity after Immune Checkpoint inhibitor therapy in Melanoma. Natural progression and Management. Am J Clin Oncol. 2017;41:760-765.
16. Friedman CF, Proverbs-Singh TA, Postow MA. Treatment of the immune-related adverse effects of immune checkpoint inhibitors: a review. JAMA Oncol. 2016;2:1346-1353.

17. Kleiner DE, Berman D. Pathologic changes in Ipilimumab-related hepatitis in patients with metastatic melanoma. Dig Dis Sci. 2012;57:2233-2240.

18. DeMartin E, Michot JM, Papouin B, et al. Characterization of liver injury induced by cancer immunotherapy using immune checkpoint inhibitors. J Hepatology. 2018;68:81-90.

19. Zen Y, Yeh MM. Hepatotoxicity of immune checkpoint inhibitors: a histology study of seven cases in comparison with autoimmune hepatitis and idiosyncratic drug induced liver injury. Mod Pathol. 2017;31:965-973.

20. Rockey DC, Seeff LB, Rochon J, et al. Causality assessment in drug-induced liver injury using a structured expert opinion process: comparison to the Roussel-Uclaf Assessment method. Hepatology. 2010;51:2117-2126.

21. Johncilla M, Misdraji J, Pratt DS, et al. Ipilimumab-associated hepatitis: clinicopathologic characterization in a series of 11 cases. Am J Surg Pathol. 2015;39:1075-1084.

22. Wang P-F, Chen Y, Song S-Y, et al. Immune-related adverse events associated with anti-PD-1/ PD-L1 treatment for malignancies: a meta-analysis. Front Pharmacol. 2017;8:730-740.

\section{SUPPORTING INFORMATION}

Additional supporting information will be found online in the Supporting Information section at the end of the article.

How to cite this article: Tsung I, Dolan R, Lao CD, et al. Liver injury is most commonly due to hepatic metastases rather than drug hepatotoxicity during pembrolizumab immunotherapy. Aliment Pharmacol Ther. 2019;50:800-808. https://doi.org/10.1111/apt.15413 